بررسى ساختار و تنوع زنتيكى در ارقام و لاينهاى كَندم ايرانى با استفاده

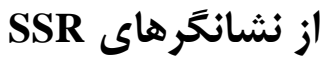

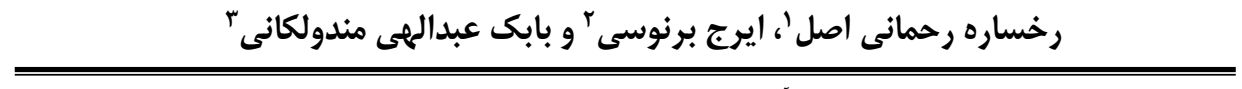 \\ ا و ب- دانشآموخته كارشناسى ارشد و دانشيار، دانشخاه اروميه

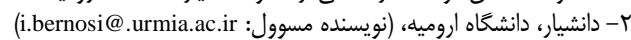

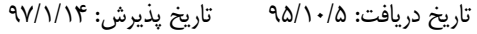

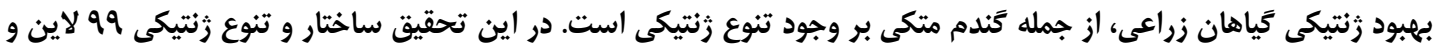

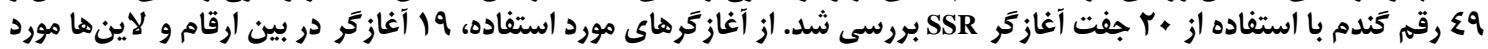

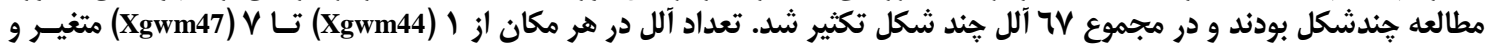

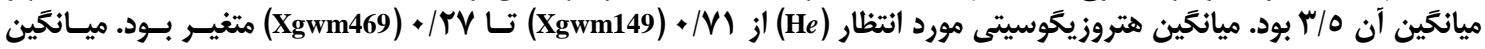

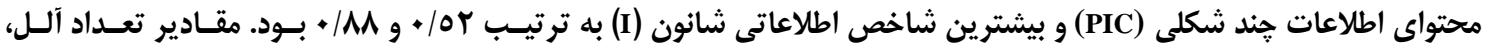

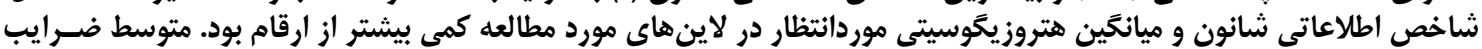

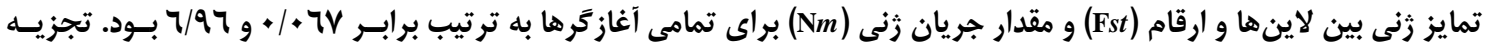

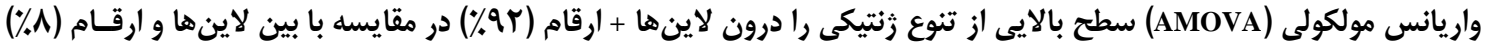

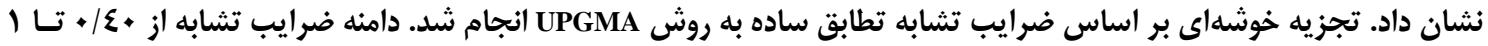

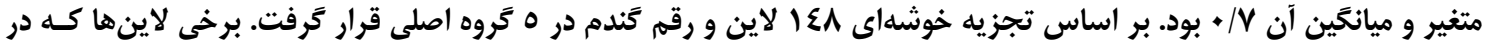

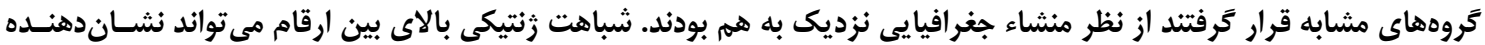

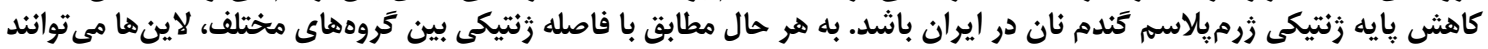

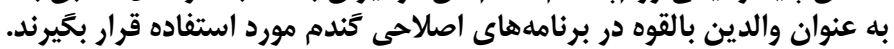

وازههاى كليدى: تَندم نان، نشانكَرهاى SSR، هتروزيخَوسيتى موردانتظار، شاخص اطلاعاتى شانون، محتواى اطلاعات جند شكلى

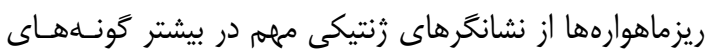

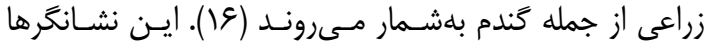

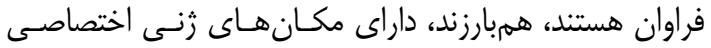

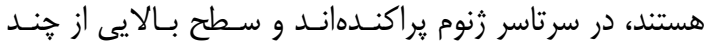

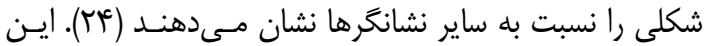

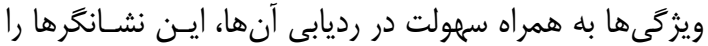

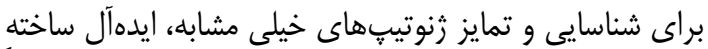

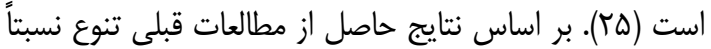

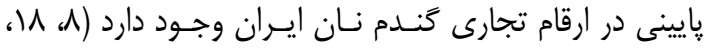

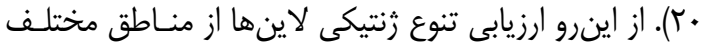

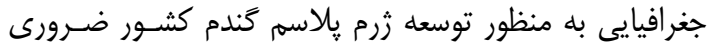

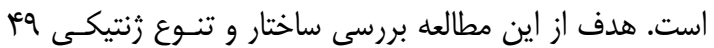

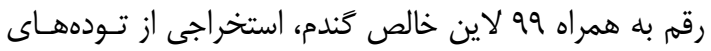

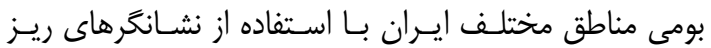

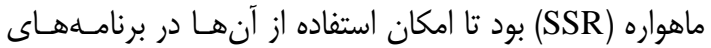
اصلاحى كندم در كشور فراهم شود.

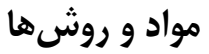

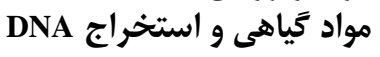

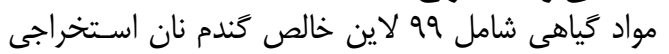

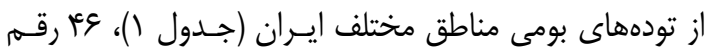

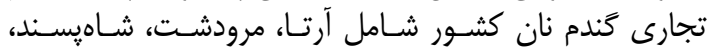

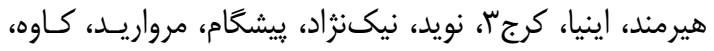

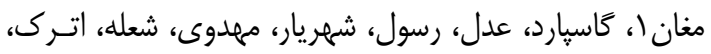

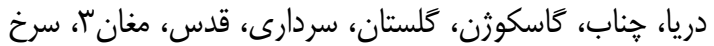

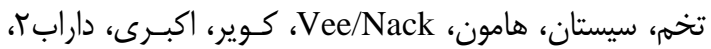

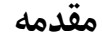

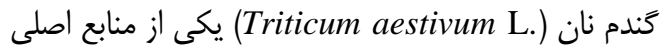

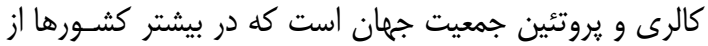

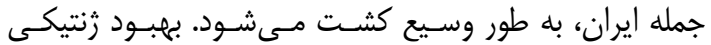

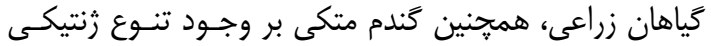

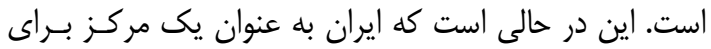

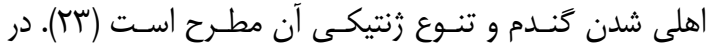

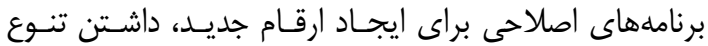

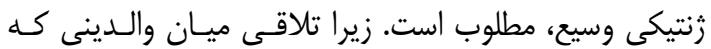

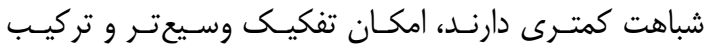

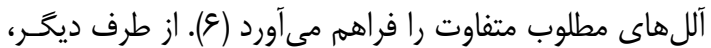

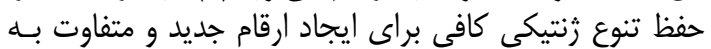

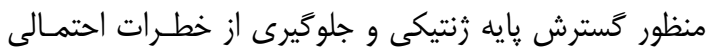

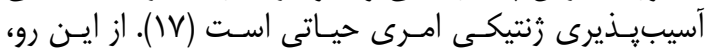

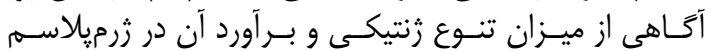

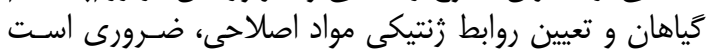

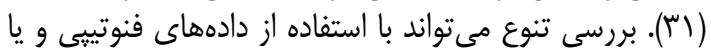

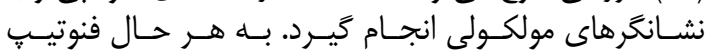

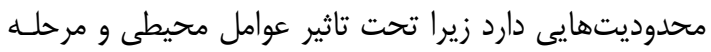

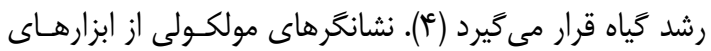

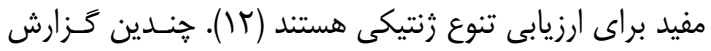

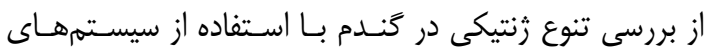

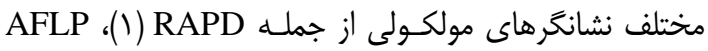

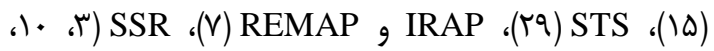

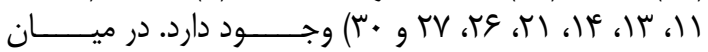
نشانكرهاى مولكولى، توالىهـاى تكـرارى سـاده (SSRs) يـان 


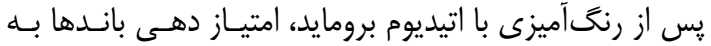

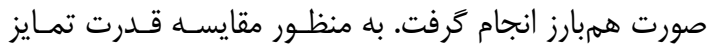

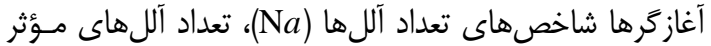

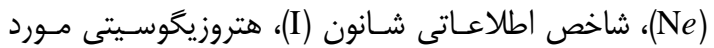

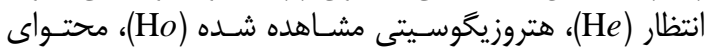

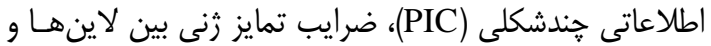

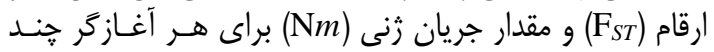

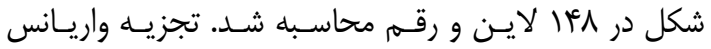

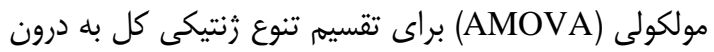

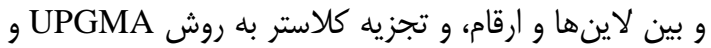

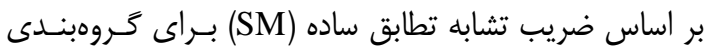

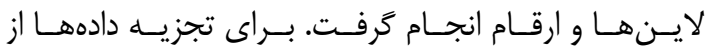

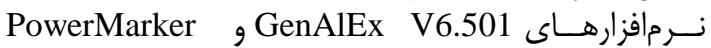
V3.25

Table 1. Origin of the studied bread wheat lines

\begin{tabular}{|c|c|c|c|c|c|c|c|c|c|c|c|}
\hline منشاء & لاين & رديف & منشاء & لاين & رديف & منشاء & لاين & رديف & منشاء & لاين & رديف \\
\hline اروميه & KC179 & ve & خوى & KC146 & DI & خوى & $\mathrm{KC} 110$ & te & تبريز & $\mathrm{KC} 1$ & 1 \\
\hline اروميه & KC180 & v & خوى & KC147 & $\Delta T$ & خوى & $\mathrm{KC} 111$ & TV & تبريز & $\mathrm{KC} 2$ & r \\
\hline اروميه & $\mathrm{KC} 183$ & $\mathrm{vA}$ & خوى & KC149 & $\Delta r$ & خوى & $\mathrm{KC} 113$ & ru & تبريز & KC5 & r \\
\hline كرمانشاه & КС608 & va & خوى & $\mathrm{KC} 151$ & $\Delta F$ & خوى & $\mathrm{KC} 114$ & rq & تبريز & KC6 & f \\
\hline كرمانشاه & КС609 & $\wedge$. & خوى & $\mathrm{KC} 152$ & $\Delta \Delta$ & خوى & $\mathrm{KC} 115$ & $r \cdot$ & تبريز & KC7 & $\Delta$ \\
\hline تربت حيدريه & $\mathrm{KC} 1080$ & 11 & خوى & $\mathrm{KC} 154$ & $\Delta \xi$ & خوى & $\mathrm{KC} 116$ & r & تبريز & $\mathrm{KC} 8$ & 9 \\
\hline تربت جام & KC1082 & Nr & خوى & $\mathrm{KC} 155$ & $\Delta V$ & خوى & $\mathrm{KC} 117$ & rr & تبريز & $\mathrm{KC} 11$ & v \\
\hline كرمانشاه & $\mathrm{KC} 1544$ & ^ & خوى & KC156 & $\Delta \Lambda$ & خوى & KC120 & זr & تبريز & $\mathrm{KC} 12$ & $\wedge$ \\
\hline كرمانشاه & KC1545 & NF & خوى & $\mathrm{KC} 157$ & $\Delta 9$ & خوى & $\mathrm{KC} 121$ & re & تبريز & $\mathrm{KC} 13$ & 9 \\
\hline كرمانشاه & $\mathrm{KC} 1546$ & $\Lambda \Delta$ & خوى & $\mathrm{KC} 158$ & 9. & خوى & $\mathrm{KC} 122$ & ro & تبريز & $\mathrm{KC} 15$ & 1. \\
\hline كرمانشاه & $\mathrm{KC} 1547$ & N & خوى & KC159 & 8) & خوى & $\mathrm{KC} 125$ & פצ & تبريز & KC16 & 11 \\
\hline كرمانشاه & KC1548 & $\wedge V$ & خوى & KC161 & gr & خوى & KC126 & rV & تبريز & KC18 & ir \\
\hline كرمانشاه & KC1549 & $M$ & خوى & KC164 & r & خوى & $\mathrm{KC} 128$ & ऍی & تبريز & KC19 & r \\
\hline كرمانشاه & $\mathrm{KC} 1550$ & 19 & خوى & KC165 & gt & خوى & $\mathrm{KC} 129$ & rq & تبريز & $\mathrm{KC} 20$ & $1 f$ \\
\hline كرمانشاه & KC1661 & q. & خوى & KC167 & $9 \Delta$ & خوى & $\mathrm{KC} 130$ & r. & تبريز & $\mathrm{KC} 21$ & 10 \\
\hline كرمانشاه & KC1662 & 91 & خوى & KC168 & 98 & خوى & KC131 & il & تبريز & $\mathrm{KC} 22$ & 19 \\
\hline سبزوار & $\mathrm{KC} 1711$ & qर & خوى & KC169 & $9 V$ & خوى & KC132 & et & تبريز & $\mathrm{KC} 23$ & IV \\
\hline سبزوار & $\mathrm{KC} 1713$ & १५ & خوى & $\mathrm{KC} 170$ & 91 & خوى & KC133 & r & تبريز & $\mathrm{KC} 25$ & 11 \\
\hline سبزوار & $\mathrm{KC} 1717$ & 94 & خوى & $\mathrm{KC} 171$ & 99 & خوى & $\mathrm{KC} 136$ & fer & تبريز & $\mathrm{KC} 26$ & 19 \\
\hline سبزوار & $\mathrm{KC} 1720$ & 90 & خوى & $\mathrm{KC} 172$ & v. & خوى & KC137 & ED & تبريز & $\mathrm{KC} 27$ & $r$. \\
\hline سبزوار & $\mathrm{KC} 1721$ & १९ & خوى & KC173 & n & خوى & $\mathrm{KC} 138$ & is & تبريز & $\mathrm{KC} 28$ & rI \\
\hline سبزوار & $\mathrm{KC} 1723$ & १V & خوى & $\mathrm{KC} 174$ & vr & خوى & KC139 & iv & تبريز & KC29 & tr \\
\hline سبزوار & $\mathrm{KC} 1724$ & 91 & خوى & $\mathrm{KC} 175$ & $v^{r}$ & خوى & $\mathrm{KC} 140$ & is & تبريز & KC30 & r \\
\hline \multirow[t]{2}{*}{ مشهد } & KC1726 & 99 & اروميه & KC177 & $v^{e}$ & خوى & KC142 & 19 & تبريز & KC31 & Mr \\
\hline & & & اروميه & $\mathrm{KC} 178$ & VD & خوى & KC145 & $\Delta$. & تبريز & $\mathrm{KC} 32$ & ra \\
\hline
\end{tabular}

بيات، به17، كرج،، روشن، آزادى، الموت، دز، ييشتاز، زرين، شيراز،

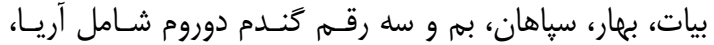

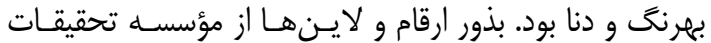

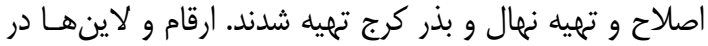

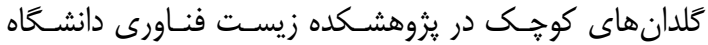

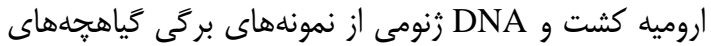
ك-1 روزه به روش

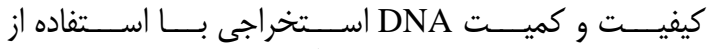

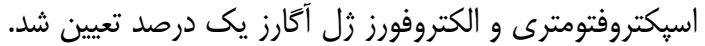
واكنث هاى SSR و تجزيه دادهنها

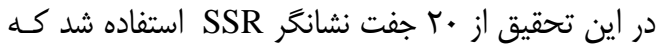

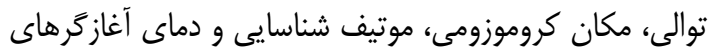

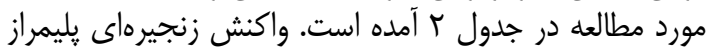

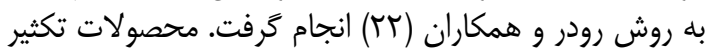

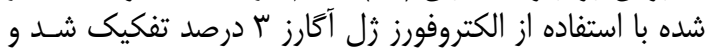

جدول ا- منشاء لاينهاى كندم نان مورد مطالعه 
شده از شهرستانهاى كرمانشاه، سبزوار و اروميه بله صورت

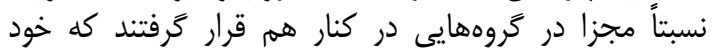

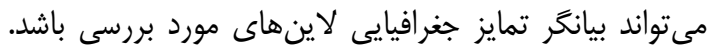

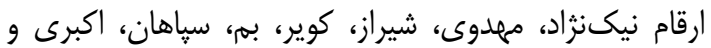

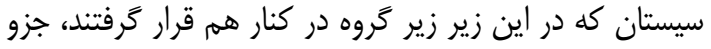

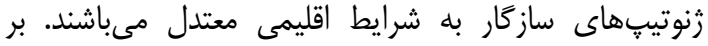

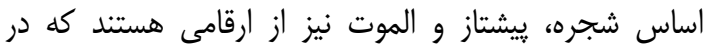

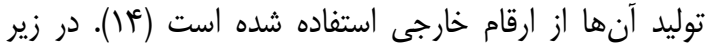

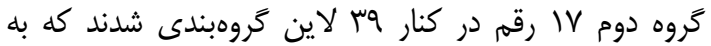

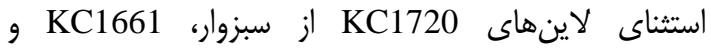

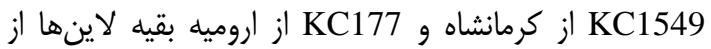

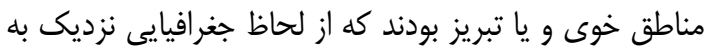

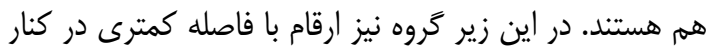

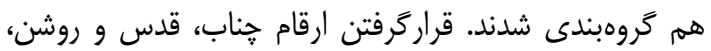

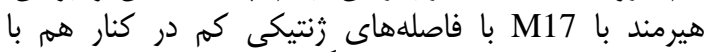

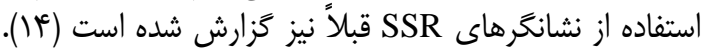

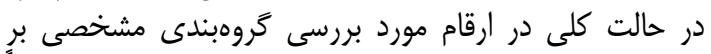

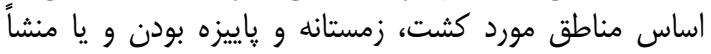

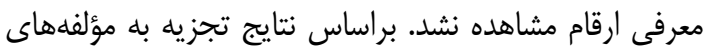

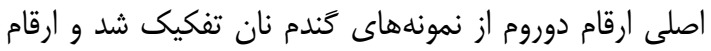

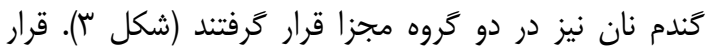

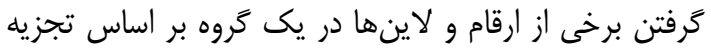

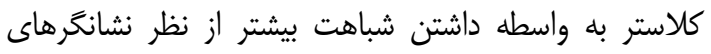
SSR

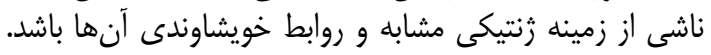

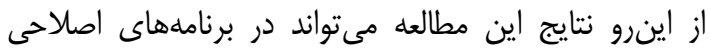

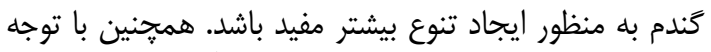

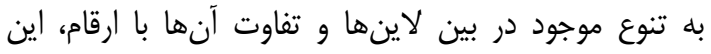

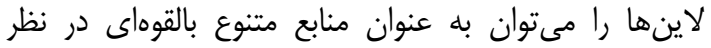

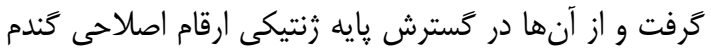

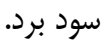

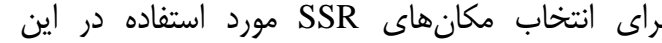

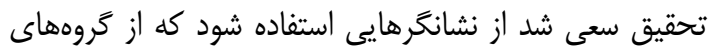

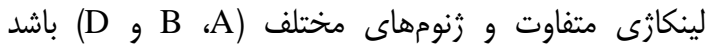

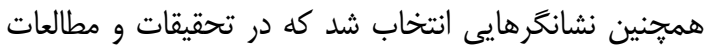

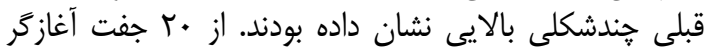
أغازكا SSR

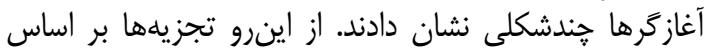
19 جفت آغازگ 19

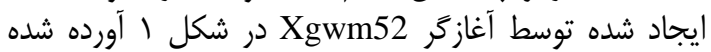

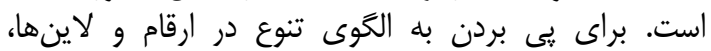

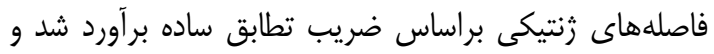

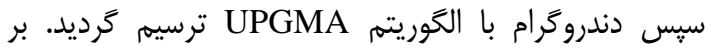

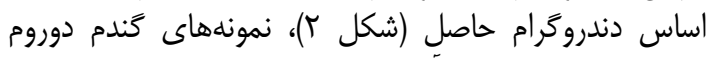

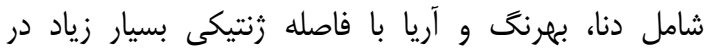

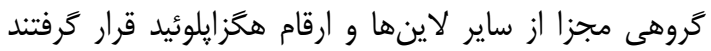

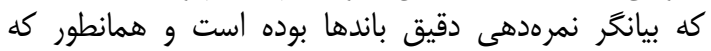

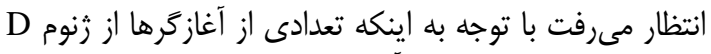

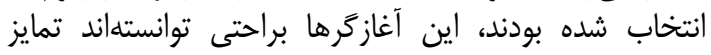

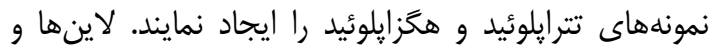

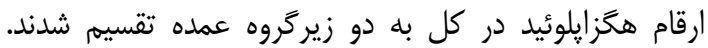

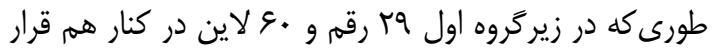

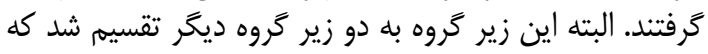

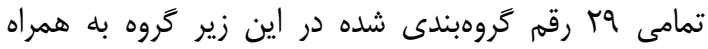
لاينهاى KC175 از شهرستان خوى، لاينهاى KC178 و KC183

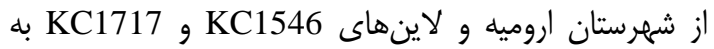

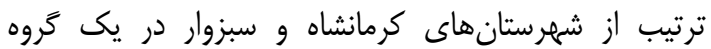

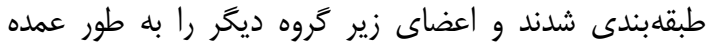

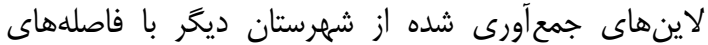

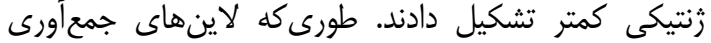

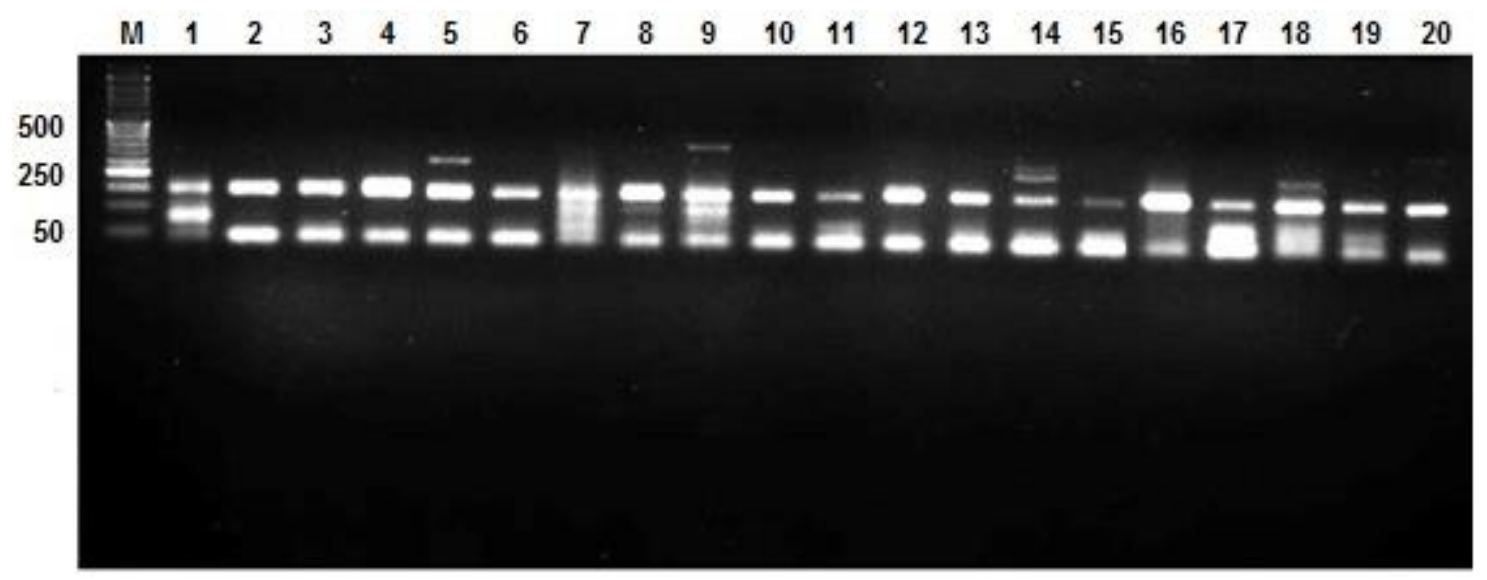

شكل 1- الكوى باندى مربوط به آغازگ

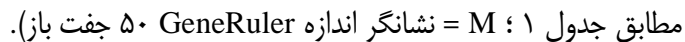

Figure 1. Banding patterns amplified by Xgwm52 primer for 20 wheat lines (Genotypes numbers 7, 8, 11, 12, 13, 15 , $16,18-23,25-31$ as in table $1 ; M=$ GeneRuler 50 bp DNA Ladder). 
جدول r- آغازگًر، توالى، مكان هاى كروموزومى، موتيف و دماى اتصال نشانخر هاى SSR

Table 2. Primer, sequence, chromosomal locations, motif identify and annealing temperature of SSR markers

\begin{tabular}{|c|c|c|c|c|c|c|}
\hline دماى اتصال & موتيف شناسايى & دامنه باندى (bp) & مكان كروموزومى & معكوس ('3'3') & مستقيم ('3'> & آغازمكر \\
\hline 9. & (AT)19(GT)15 & $199-r \cdot \Delta$ & $2 \mathrm{D}, 3 \mathrm{~A}$ & $\begin{array}{l}\text { TAGTGGTCCCACGTTT } \\
\end{array}$ & $\begin{array}{l}\text { ATCTTAGCATAGAAGGGAGTGGG } \\
\end{array}$ & Xgwm30 \\
\hline 9. & (CA) 22 & IVG-IAF & $7 \mathrm{~B}$ & GTGTACTGTAAACCGTGAGTGG & CACCGACGGTTTCCCTAGAGT & $\mathrm{Xgwm} 43$ \\
\hline 9. & $(\mathrm{GA}) 28$ & $I V Q-I V A$ & 7D & GTCGAGACACCTACGGTCA & GTTGAGCTTTTCAGTTCGGC & $\mathrm{Xgwm} 44$ \\
\hline 9. & (CT)7TT(CT)16 & $1 Q D-1 M$ & $2 \mathrm{~A}, 2 \mathrm{~B}$ & TCCTGGAGTTAGCTCCACTT & TTGCTACCATGCATGACCAT & $\mathrm{Xgwm} 47$ \\
\hline 9. & (GT)4AT(GT)20 & ITA-IFT & 3D & TTTACCTTCTCGTGGCGT & CTATGAGGCGGAGGTTGAAG & $\mathrm{Xgwm52}$ \\
\hline 4. & (AC) 16 & $\| \varphi-1 T \Lambda$ & $2 \mathrm{~A}$ & GCCCAAAAAGTGAATCGTAA & GATCAAACACACAССССТCC & Xgwm95 \\
\hline 9. & (CT) 58 & $T V \Lambda-M T I$ & $1 \mathrm{~A}$ & СТACTCGTACAACGGCTAC & GACAGCACCTTGCCCTTTG & Xgwm136 \\
\hline$\Delta \Delta$ & (GA) 23 & $|Q T-| 9 \mid$ & $4 \mathrm{~B}$ & GAACAAGTCCAAGCTACGATC & CATTGTTTTCTGCCTCTAGCC & Xgwm149 \\
\hline 4. & $(\mathrm{GA}) 18$ & $1 A F-190$ & 1B & GAGAGGCAGGAAGAGATGGT & GATCTCGTCACCCGGAATTC & Xgwm153 \\
\hline 9. & (GA)21 & $145-195$ & $4 \mathrm{~A}$ & CCACATGAAAAAAAGGACGTC & TTCAATTCAGTCTTGGCTTGG & Xgwm160 \\
\hline 4. & (GA) 20 & $|M-r q|$ & $4 \mathrm{~A}, 4 \mathrm{D}, 4 \mathrm{~B}$ & CCGCGTTAGACTTTCTTTTC & TGCAGTGGTCAGAGTTTTCC & Xgwm165 \\
\hline c. & (CT) 18 & $\mid \mathcal{Q H - I A V}$ & 5D & GGAATAAACCGACACACGTT & TGATGTAGTGAGCCCATAGGC & Xgwm182 \\
\hline 4. & (CT) 19 & $1 \cdot V-I Y F^{\prime}$ & $6 \mathrm{~B}, 5 \mathrm{~B}$ & GTACGTATGTTGACAGCACGAT & AGACTGTTGTTTGCGGGC & Xgwm191 \\
\hline$\Delta \Delta$ & $(\mathrm{GA}) 11(\mathrm{GGA}) 8$ & $10 \cdot-1 \Lambda$ & $2 \mathrm{~A}, 2 \mathrm{D}$ & CCATCTAGGTCTTTTTACCGTC & CAAATGGATCGAGAAAGGGA & Xgwm249 \\
\hline 9. & $(\mathrm{GA}) 36$ & rII-rq. & $7 \mathrm{~A}$ & CGAAGTGATGAGAAAGGTCGTGA & AGCCAGCAAGTCACCAAAAC & Xgwm332 \\
\hline$\Delta \Delta$ & (CT) 5 (СACT)6(CA) 43 & $|A T-19|$ & 1D & CCGTTTCCGGTCAATCGT & ССТСТTССТСССТСАСТTAGC & Xgwm337 \\
\hline$\Delta \Delta$ & (CA)22(TA)(CA)7(TA)9 & $\| \& \Lambda-I \Lambda T$ & $5 \mathrm{~B}$ & GCACGACACTTGCTTAATATG & TCGATTTATTTGGGCCACTG & Xgwm 408 \\
\hline a. & (CA) $31(\mathrm{CA}) 22$ & $189-190$ & $6 \mathrm{~A}$ & TTGACAGTTTACTTGTGTGA & AAACTTAGAACTGTAATTTCAGA & Xgwm427 \\
\hline 4. & (CT) $19(\mathrm{CA}) 10$ & $I V \cdot-I V T$ & $6 \mathrm{D}$ & ССАСАССТАСТСАССАATAGC & CAACTCAGTGCTCACACAACG & Xgwm469 \\
\hline 9. & (CT) $18(\mathrm{CA}) 20$ & $|r \cdot-\mu| S$ & 3B & CCAAAAGGGGATTTCGTTG & AAGGCGAATCAAACGGAATA & Xgwm533 \\
\hline
\end{tabular}




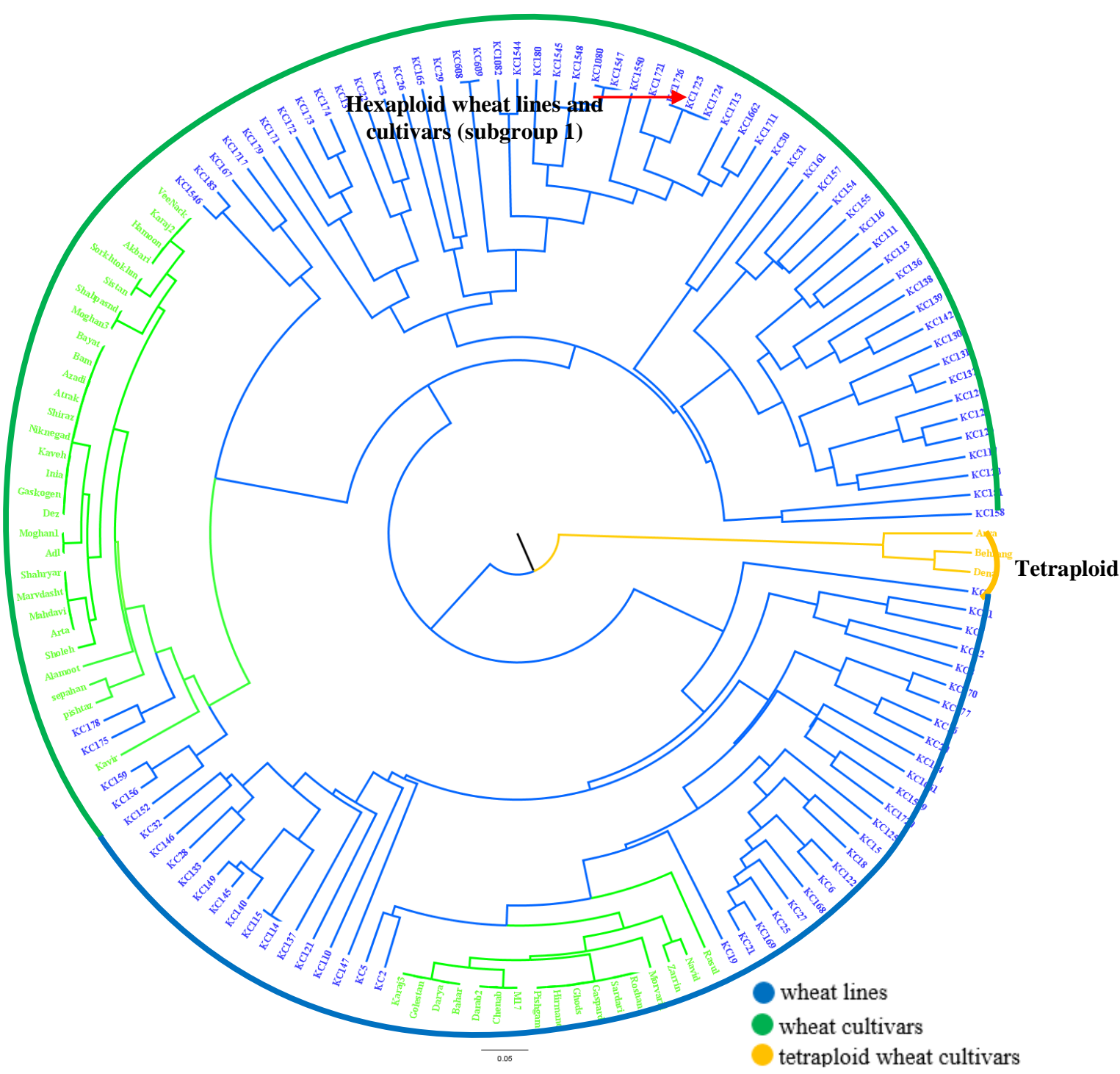

Hexaploid wheat lines and cultivars (subgroup 2)

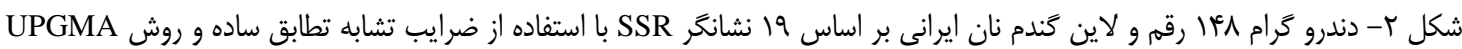
Figure 2. Dendrogram of 148 Iranian bread wheat lines and cultivars using 19 SSR markers based on simple matching coefficients and UPGMA methods. 


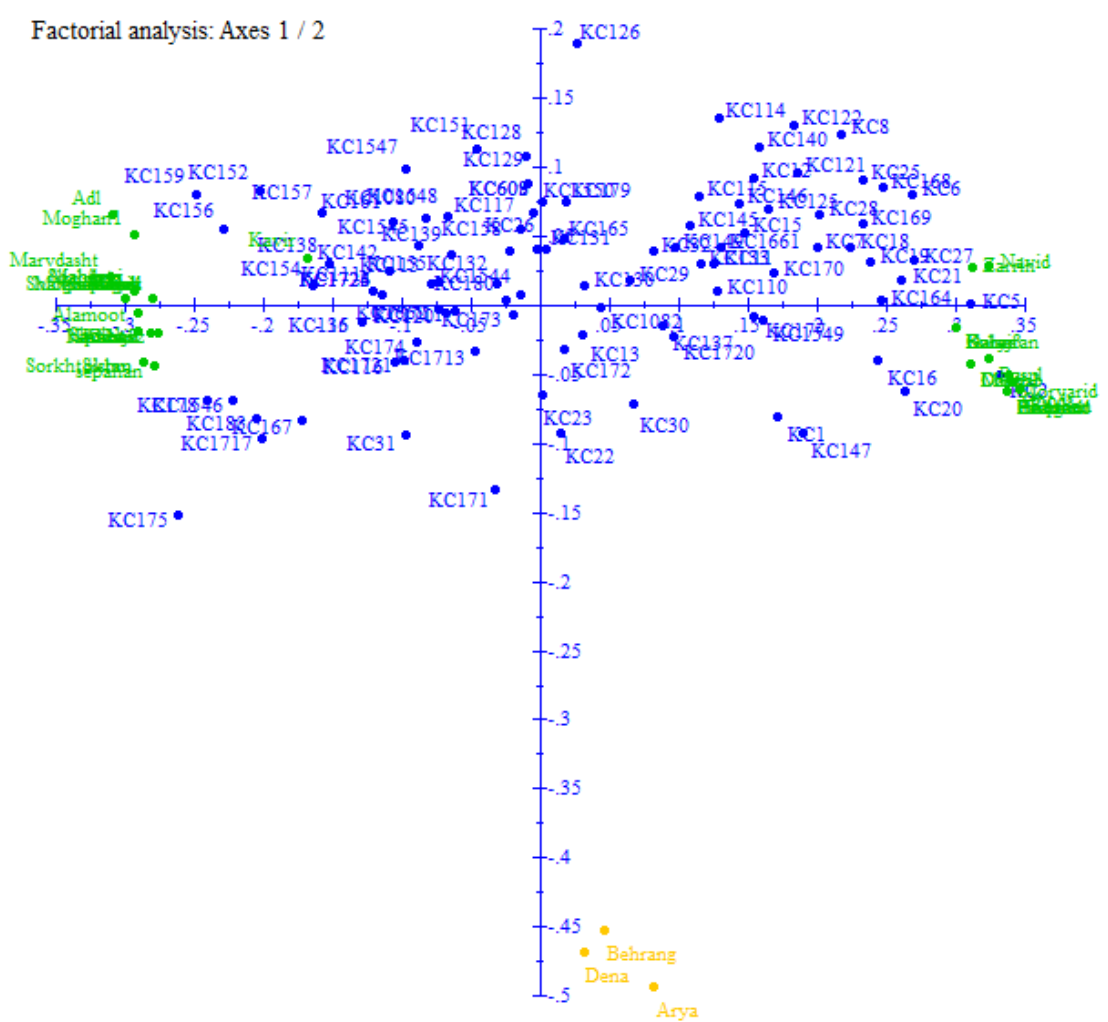

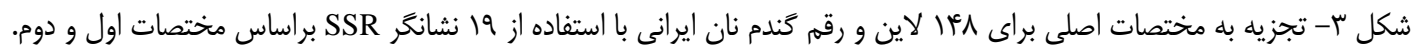
Figure 3. Principal coordinate analysis for 148 Iranian bread wheat lines and cultivars using 19 SSR markers based on first and second coordinates.

متفاوت را فراهم مىآورد. براى يك تعداد آلل معين، بيشترين

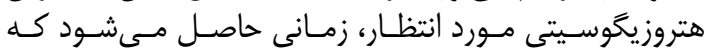

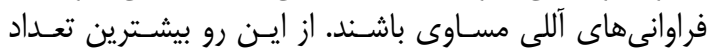

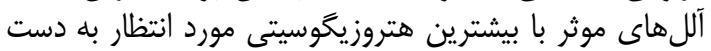

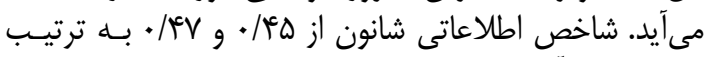

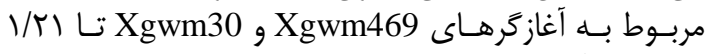

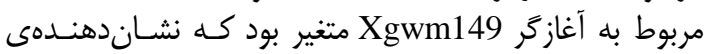

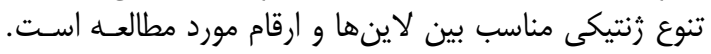

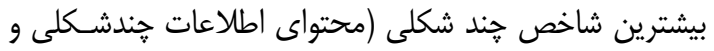

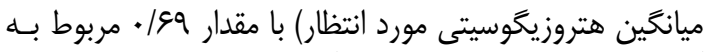

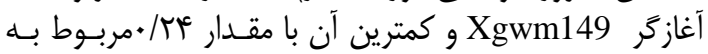

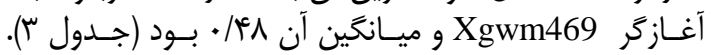

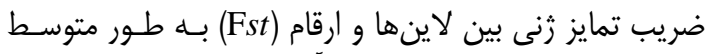

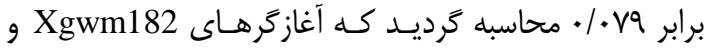

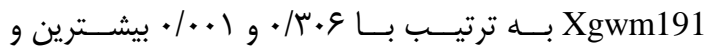

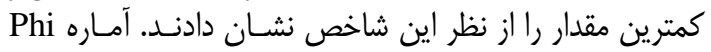

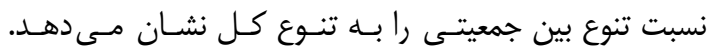

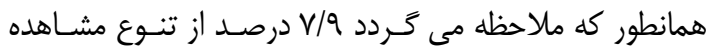

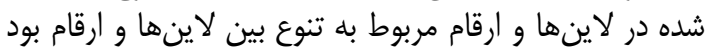

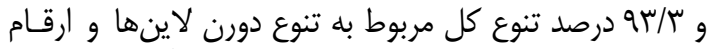

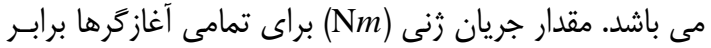

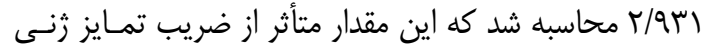

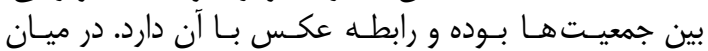

در ادامه براى تجزيه هــاى مـرتبط بــا تنـوع آللـى، تمـايز

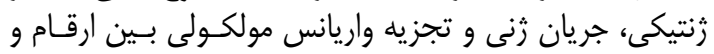

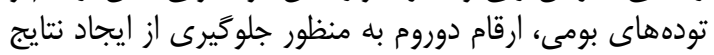

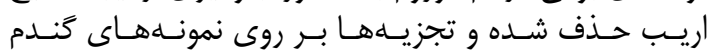

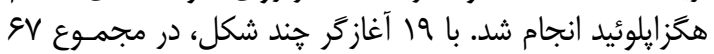

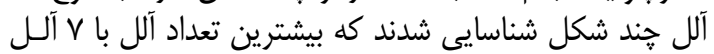

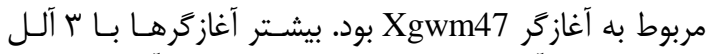

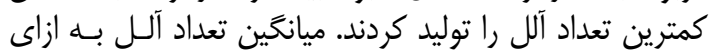

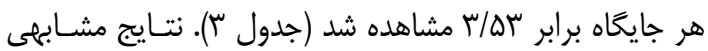

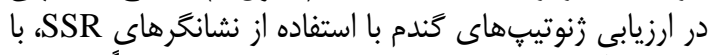

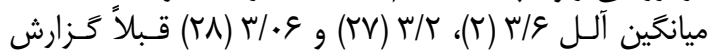

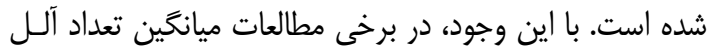

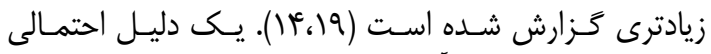

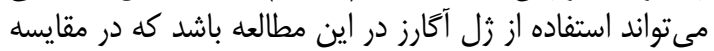

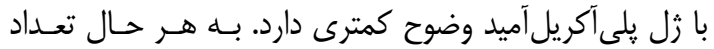

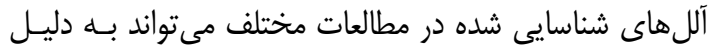

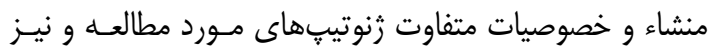

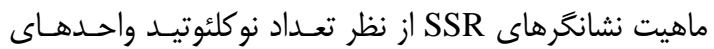

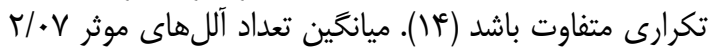

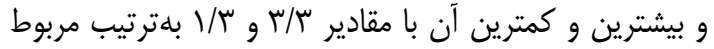

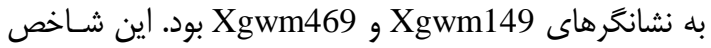

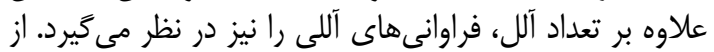

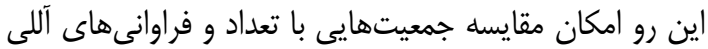




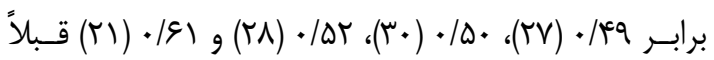

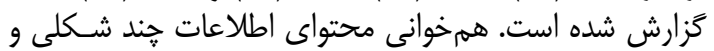

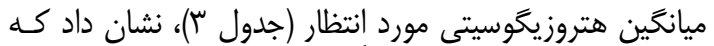

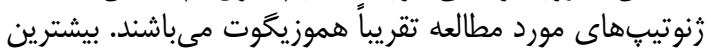

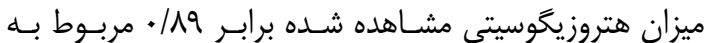

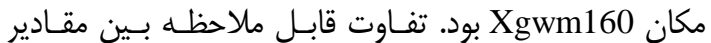

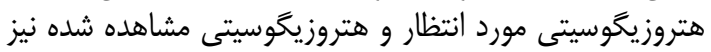
حاكى از عدم وجود تعادل هاردى واينبرى است.
آغاز كرهاى مورد بررسى نيز بيشترين جريان رُنى بين لاينهـا

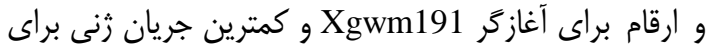

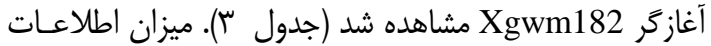

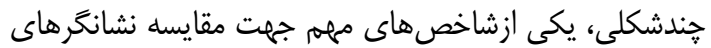

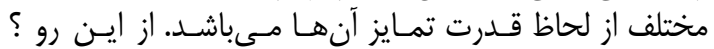

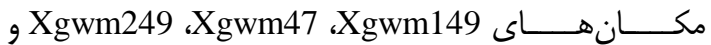

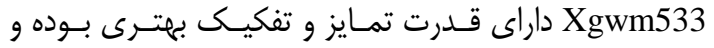

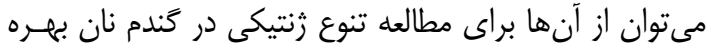

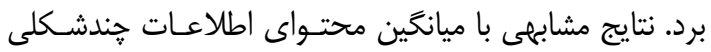

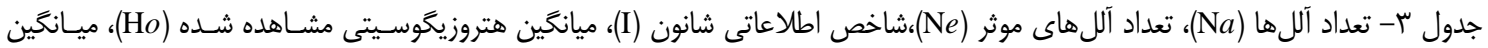

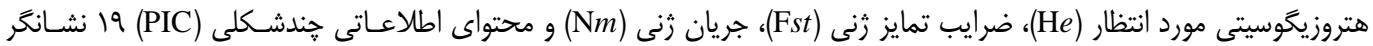
SSR

Table 3. Number of alleles ( $\mathrm{N} a$ ), effective number of alleles ( $\mathrm{N} e$ ), Shannon's information index (I), Mean observed heterozygosity $(\mathrm{Ho})$, Mean expected heterozygosity $(\mathrm{H} e)$, coefficients of gene differentiation (Fst), gene flow $(\mathrm{N} m)$ and Polymorphism information content (PIC) of the 19 SSR markers applied to wheat lines and cultivars

\begin{tabular}{|c|c|c|c|c|c|c|c|c|c|}
\hline PIC & $\mathrm{Nm}$ & Fst & $\mathrm{He}$ & Hо & $I$ & $\mathrm{Ne}$ & $\mathrm{Na}$ & تعداد & نام نشانگر \\
\hline$\cdot / K F$ & $|/ 94|$ & •/Nץ*** & $\cdot / \mu \cdot \pm \cdot / / \mu$ & $\cdot / f \backslash \pm \cdot / T r$ & $\cdot / \& \vee \pm \cdot / \backslash q$ & $1 / \Delta \pm \cdot / / \Lambda$ & $r / \cdot \pm \cdot / \cdot$ & $r$ & Xgwm30 \\
\hline- & - & - & - & - & - & - & - & - & Xgwm44 \\
\hline$\cdot / \Delta V$ & 11/^9У & $\cdot / \cdot r \mid *$ & $\cdot / \Delta q \pm \cdot 1 \cdot{ }^{f}$ &.$/ 1 V_{ \pm} \cdot 1 \cdot 9$ & $1 / / F_{ \pm} \cdot / 10$ & $T / T_{ \pm} \cdot / T F$ & $\Delta / \Delta \pm 1 / \Delta$ & v & Xgwm47 \\
\hline$\cdot|\Delta|$ & $1 / 90$ & $\cdot / 11 千 * *$ & $\cdot / \& q \pm \cdot / / f$ & . $198 \pm \cdot / 4 T$ & שTI/RTE & $r / I \pm \cdot / \Delta q$ & $\mu / . \pm \cdot /$. & r & Xgwm136 \\
\hline .190 & $119 / 9 \mathrm{~V}$ & $\cdot / \cdot r n s$ & . $/ q_{ \pm \pm \cdot}$ & $\cdot / 0 \cdot \pm \cdot / 11$ & $1 / 4 \pm=/ 14$ & سא/• & $\Gamma / \Delta \pm \cdot / \Delta$ & f & Xgwm149 \\
\hline . & s/ord & $\cdot / \cdot r V * *$ & $\cdot / \Delta r_{ \pm+1 \cdot \varphi}$ & $\cdot / \mu \cdot \pm \cdot / \mu \cdot$ & $\cdot / \Lambda \varepsilon_{ \pm \cdot / / \Lambda}$ & $r / T \pm \cdot / r \Lambda$ & $\Psi / \cdot \pm 1 /$. & r & Xgwm153 \\
\hline$\cdot / \Delta$ & r/rgY & $.1 .99 * *$ & $\cdot / \Delta V \pm \cdot / \cdot \Delta$ & $\cdot / 19 \pm \cdot / 11$ &.$/ 9 u_{ \pm} \cdot 1.9$ & $r / \tau_{ \pm} \cdot / r \varepsilon$ & $r / \cdot \pm • /$. & r & Xgwm160 \\
\hline$\cdot / \uparrow \wedge$ & $1 / A \cdot r$ & ./rY*** & $\cdot / \& \Delta \pm \cdot / / \Delta$ & $\cdot /\left.g\right|^{c} \pm \cdot / T V$ & $\cdot / V^{E \pm} \pm / T q$ & $r / \cdot \pm \cdot / \Delta r$ & $r / \Delta \pm \cdot / \Delta$ & r & Xgwm332 \\
\hline$\cdot / 4 V$ & $1 / \cdot \Delta 1$ &.$/ 19 Y^{* * *}$ & $\cdot /<q \pm \cdot / \cdot r$ & $\cdot / \cdot \pm \cdot / \cdots$ & $\cdot / v \varepsilon_{ \pm} \cdot / 1 I$ & $r / \cdot \pm \cdot / \cdot \Lambda$ & $\Gamma / \Delta \pm r / \Delta$ & r & Xgwm337 \\
\hline . $/ 4 q$ & $1 / T F$. &.$/ ノ \wedge^{* * *}$ & س & $\cdot / \cdot \pm+/ \cdot$ & $\cdot / \Delta \Delta \pm \cdot / 11$ & $1 / \varepsilon_{ \pm} \cdot / \mu$ & $\Gamma / \Delta \pm \cdot / \Delta$ & r & Xgwm408 \\
\hline$\cdot / 4 t$ & 10/9T9 & $\cdot / \cdot 10^{*}$ & $\cdot / \Delta T \pm \cdot / \cdot \Delta$ & rו/ & $\cdot / V \wedge \pm \cdot / 1 T$ & $r / \Lambda \pm \cdot / r)$ & $\Gamma / \Delta \pm \cdot / \Delta$ & r & Xgwm427 \\
\hline •/TD & V/IR & . /.rץt** & $\cdot / \mu_{ \pm \pm}^{c} \cdot / \cdot \Lambda$ & $\cdot / r q \pm \cdot / 11$ & $\cdot / \& \Delta \pm \cdot / 1 \Delta$ & $1 / \mu_{ \pm} \cdot / / Q$ & $\Gamma / \Delta \pm 1 / \Delta$ & $\Delta$ & Xgwm469 \\
\hline$\cdot / \Delta F$ & $1.1 . v$. & $\cdot / \cdot r f *$ & $\cdot / 8 \cdot \pm \cdot 1 \cdot 4$ & $\cdot / \Lambda \Delta \pm \cdot / / \Delta$ & $V \cdot r \pm \cdot / r$ & $r / \Delta \pm \cdot / r r$ & $\mid / \cdot \pm 1 / \cdot$ & $\Delta$ & Xgwm533 \\
\hline . $/ 4 q$ & r/quा &.$/ . v 9 * *$ & $\cdot / 4 \wedge \pm \cdot / \cdot r$ & $. / 4 \Psi \pm \cdot / \cdot q$ & $\cdot / q_{ \pm} \cdot / \cdot f$ & $r / \cdot V \pm \cdot / / V$ & $r / q T \pm \cdot / I V$ & س/ه & Mean \\
\hline
\end{tabular}

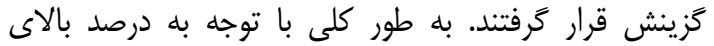

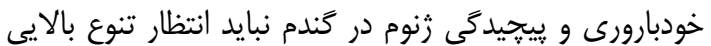

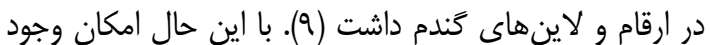

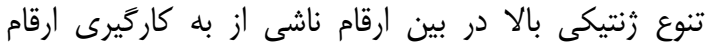

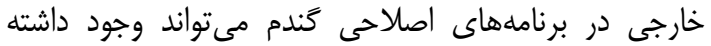

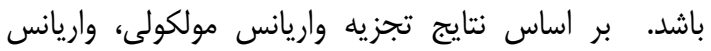

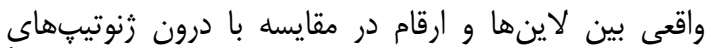

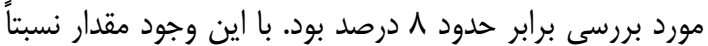

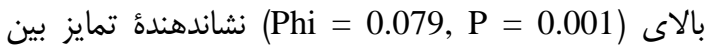
لاينها و ارقام مىباشد (جدول ه).

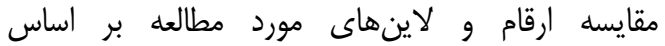

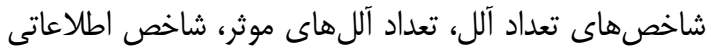

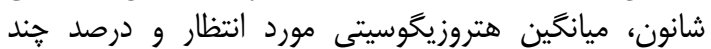

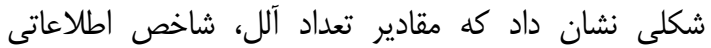

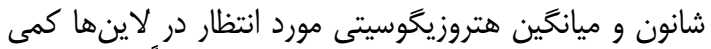

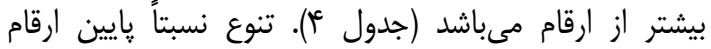

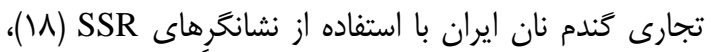
و IRAP و REMAP) ISSR است. اين مىتواند به دليل استفاده مداوم از برخى ارقام إنام باريز

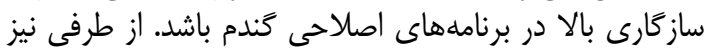

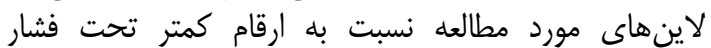


أغازگر SSR مورد استفاده در اين تحقيق تمام الب كروموزوم

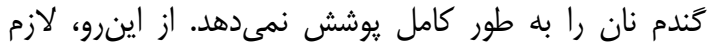

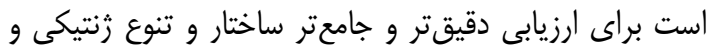

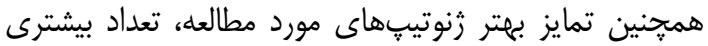

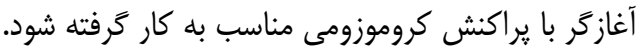

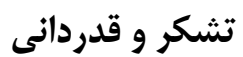

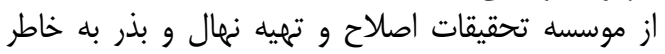

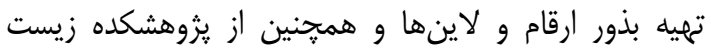

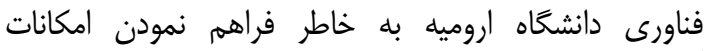

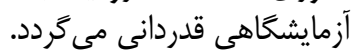

نتايج تحقيق حاضر حاكى از كارآمدى نشانغرهاى

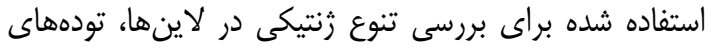

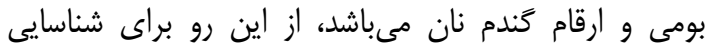

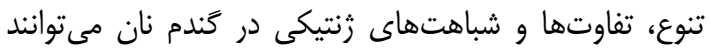

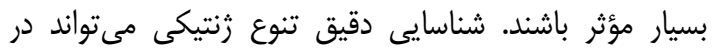

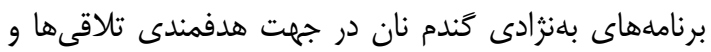

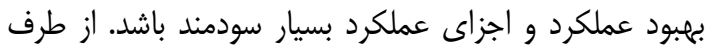

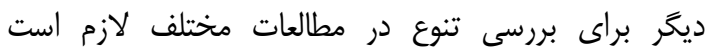

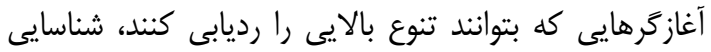

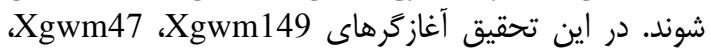

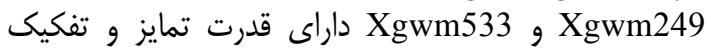

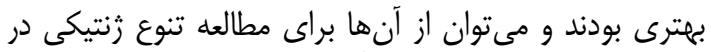

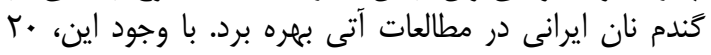

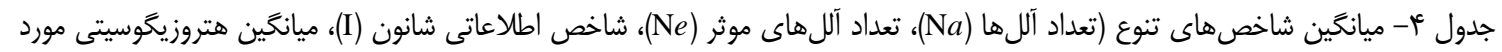

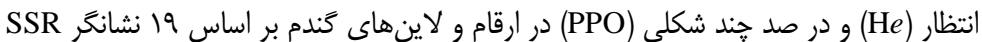

Table 4. The mean of diversity indices (number of alleles (Na), effective number of alleles (Ne), Shannon's information index (I), mean expected heterozygosity (He) and percentage of polymorphism (PPO) in wheat lines and cultivars based on 19 SSR markers

\begin{tabular}{|c|c|c|c|c|c|}
\hline PPO & $\mathrm{He}$ & I & $\mathrm{N} e$ & $\mathrm{~N} a$ & كروها \\
\hline $1 .$. & $\cdot|\Delta| \cdot \pm \cdot / \cdot r$ & $\cdot \mid \wedge \varepsilon q_{ \pm} \cdot 1 \cdot 9$ & $T / T \cdot \pm \cdot / T f$ & $T / \mathbb{E T}^{\prime} \cdot / T V$ & لاينها \\
\hline $1 .$. & $\cdot / \leftarrow \Delta \wedge \pm \cdot / \cdot \mu$ & $\cdot / v \cdot \Lambda \pm \cdot / \cdot \Delta$ & $1 / 9 q_{ \pm} \cdot / 1$. & $r / F T \pm \cdot / R$ & ارقام \\
\hline
\end{tabular}

جدول ه- تجزيه واريانس مولكولى بين هأl لاين و رقم كندم نان ايرانى براساس 19 نشانگر (AMOVA)

Table 5. Analysis of molecular variance (AMOVA) for 145 Iranian bread wheat lines and cultivars using 19 SSR markers

\begin{tabular}{|c|c|c|c|c|c|c|}
\hline مقدار احتمال & (Phi) F آماره & در صد & واريانس برآورد شده & ميانكين مربعات & درجه آزادى & منبع تغييرات \\
\hline \multirow[t]{4}{*}{.$/ .1$} & $.1 . v 9$ & $V / \Lambda S$ &.$/ F T$. & 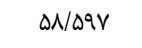 & 1 & بين جمعيتها (لاينها و ارقام) \\
\hline & & $I V / 1 Q$ &.$/ 919$ & $\Delta / \wedge r q$ & سer & بين افراد درون جمعيتها \\
\hline & & $V \varepsilon / 99$ & $r / \cdot . V$ & $r / . . v$ & IFD & درون افراد \\
\hline & & $1 \ldots$ & W/T/W & D/TFY & r/q & كل \\
\hline
\end{tabular}

1. Abdollahi Mandoulakani, B., A.A. Shahnejat-Bushehri, B.E. Sayed Tabatabaei, S. Torabi and A. Mohammadi Hajiabad. 2010. Genetic diversity among wheat cultivars using molecular markers. Journal of Crop Improvement, 24: 299-309.

2. Ahmed, M. 2002. Assessment of genomic diversity among wheat genotypes as determined by simple sequence repeats. Genome, 45: 646-651.

3. Akkaya, M.S. and E.B. Buykunal-Bal. 2004. Assessment of genetic variation of bread wheat varieties using microsatellite Euphytica, 135: 179-185.

4. Archak, S., A.B. Gaikwad, D. Gautam, E.V. Rao, K.R. Swamy, and J.L. Karihaloo. 2003. Comparative assessment of DNA fingerprinting techniques (RAPD, ISSR and AFLP) for genetic analysis of cashew (Anacardium occidentale L.) accessions of India. Genome, 3: 362-369.

5. Ausubel, F.M., R. Brent, R.E. Kingston, D.D. Moore, J.G. Seidman, J.A. Smith, K. Struhl, L.M. Albright D.M. Coen and A. Varki. 1995. Current protocols in molecular biology. Jon Wiley, New York, $4725 \mathrm{pp}$.

6. Bered, F., J.F. Barbosa-Neto and F.I.F. de Carvalho. 2002. Genetic variability in common wheat germplasm based on coefficients of parentage. Genetic Molecular Biology, 25: 211-215.

7. Carvalho, A., H. Guedes-Pinto, P. Martin-Lopes and J. Lima-Brito. 2010. Genetic variability of old Portuguese bread wheat cultivar assayed by IRAP and REMAP markers. Annual Applied Biology, 156: 337- 345.

8. Dashchi, S., B. Abdollahi Mandoulakani, R. Darvishzadeh and I. Bernousi. 2013. Molecular similarity relationships among Iranian bread wheat cultivars and breeding lines using ISSR markers. Notulae Botanicae Horti Agrobotanici Cluj-Napoca, 40: 254-260.

9. Devos, K.M. and M.D. Gale. 1992. The use of random amplified polymorphism DNA markers in wheat. Theoretical and Applied Genetics, 84: 567-572.

10. Dreisigacker, S., P. Zhang, M.L. Warburton, B. Skovmand, D. Hoisington and A.E. Melchinger. 2005. Genetic diversity among and within CIMMYT wheat landrace accessions investigated with SSRs and implications for plant genetic resources management. Crop Science, 45: 653-661. 
11. Gorji, A.H. and M. Zolnoori. 2011. Genetic diversity in hexaploid wheat genotypes using microsatellite markers. Asian Journal of Biological Sciences, 3: 368-377.

12. Habash, D.Z., Z. Kehel and M. Nachit. 2009. Genomic approaches for designing durum wheat ready for climate change with a focus on drought. Journal of Experimental Botany, 60: 2805-2815.

13. Huang, X.Q., A. Borner, M.S. Roder and M.W. Ganal. 2002. Assessing genetic diversity of wheat (Triticum aestivum L.) germplasm using microsatellite markers. Theoretical and Applied Genetics, 105: 699-707.

14. Jamalirad, S., A. Mohammadi, M. Khodarahmi and M. Toorchi. 2008. Assessing genetic relationships of bread wheat varieties based on allelic diversity of microsatellite markers. Modern Genetics Journal, 1: $79-89$ (In Persian).

15. Khalighi, M., A. Arzani and M.M. Poursiahbidi. 2008. Assessment of genetic diversity in Triticum spp. and Aegilops spp. Using AFLP markers. African Journal of Biotechnology, 7: 546-552.

16. Ma, Z.Q., M.S. Roder and M.E. Sorrells. 1996. Frequencies and sequence characteristics of di-, tri-, and tetra nucleotide microsatellites in wheat. Genome, 39: 123-130.

17. Mir, R.R., J. Kumar, H.S. Balyan and P.K. Gupta. 2012. A study of genetic diversity among Indian bread wheat (Triticum aestivum L.) cultivars released during last100 years. Genetic Resource Crop Evolution, 59: 717-726.

18. Mohammadi, S.A., M. Khodarahmi, S. Jamalirad and M.R. Jalal Kamali. 2009. Genetic diversity in a collection of old and new bread wheat cultivars from Iran as revealed by simple sequence repeatbased analysis. Annual Applied Biology, 154: 67-76.

19. Mollaheydari Bafghi, R., A. Baghizadeh, G.H. Mohammadi-Nejad and B. Nakhoda. 2014. Assessment of genetic diversity in Iranian wheat (Triticum aestivum L.) cultivars and lines using microsatellite markers. Journal of Plant Molecular Breeding 2(1): 74-89.

20. Nasri, Sh., B. Abdollahi Mandoulakani, R. Darvishzadeh and I Bernousi. 2013. Retrotransposon insertional polymorphism in Iranian bread wheat cultivars and breeding lines revealed by IRAP and REMAP markers. Biochemical Genetics, 51: 927-943.

21. Pahlavani, S., A. Izanloo, S. Parsa and M.G. Ghaderi. 2016. Association between grain quality traits and SSR molecular markers in some bread wheat genotypes. Journal of Crop Breeding, 8(19): 25-36 (In Persian).

22. Roder, M.S., V. Korzun, K. Wendehake, J. Plaschke, M.H. Tixier, P. Leroy and M.W. Ganal. 1998. A microsatellite map of wheat. Journal Genetics Society of America, 149: 2007-2023.

23. Roussel, V., J. Koenig, M. Beckert and F. Balfourier. 2004. Molecular diversity in French bread wheat accessions related to temporal trends and breeding programs. Theoretical and Applied Genetics, 108: 920-930.

24. Saghai-Maroof M.A., R.M. Biyashev, G.P. Yang, Q.F. Zhang and A.W. Allard. 1994. Extraordinarily polymorphic microsatellite DNA in barley: Species diversity, chromosomal locations, and population dynamics. Proceedings of the National Academy of Sciences of the United States of America, 91:5466-5470.

25. Saker, M., M. Nagchtigall and T.A. Kuehne. 2005. Comparative assessment of DNA fingerprinting by RAPD, SSR and AFLP in genetic analysis of some barley genotypes. Egyptian Journal of Genetics and Cytology, 34: 81-97.

26. Sardouie-Nasab, S., G.h. Mohammadi-Nejad and B. Nakhoda. 2013. Assessing genetic diversity of promising wheat (Triticum aestivum L.) lines using microsatellite markers linked with salinity tolerance. Journal of Plant Molecular Breeding, 2: 28-39.

27. Schuster, I., E.S.N. Vieira, G.J. da Silva, F.A. Franco and V.S. Marchioro. 2009. Genetic variability in Brazilian wheat cultivars assessed by microsatellite markers. Genetic Molecular Biology, 32: 557563.

27. SenturkAkfirat, F. and A. AltinkutUncuoglu. 2013. Genetic diversity of winter wheat (Triticum aestivum L.) revealed by SSR markers. Biochemical Genetics, 51: 223-229.

29. Talbert, L.E., N.K. Blake, P.W. Chee, T.K. Blake and G.M. Magyar. 1994. Evaluation of sequencetagged-site-facilitated PCR products as molecular markers in wheat. Theoretical and Applied Genetics, 87: 789-794.

30. Zergani, M., G. Ranjbar and S. Ebrahimnezhad. 2015. Molecular assessment of genetic diversity among bread wheat (Triticum aestivum L.) doubled haploid lines using SSR markers. Journal of Crop Breeding, 7(15): 88-95 (In Persian)

31. Zhangh, D. and M.H. Godfry. 2003. Nuclear DNA analyses in genetic studies of populations: practice, problems and prospects. Molecular Ecology, 12: 563-584. 


\title{
Study of Genetic Structure and Diversity of Iranian Wheat Lines and Cultivars using SSR Markers
}

\author{
Rokhsareh Rahmani Asl ${ }^{1}$, Iraj Bernousi ${ }^{2}$ and Babak Abdollahi Mandulakani ${ }^{3}$
}

1 and 3- Graduated M.Sc. Student and Associate Professor, Urmia University

2- Associate Professor, Urmia University (Corresponding author: i.bernosi@mail.urmia.ac.ir)

Received: December 25, 2016 Accepted: April 3, 2018

\begin{abstract}
Genetic improvement of crop plants such as wheat, relies on genetic diversity. In the current investigation, the genetic diversity of 99 wheat lines and 49 cultivars were assessed using 20 SSR primers. Out of the primers used, 19 were polymorphic among studied lines and cultivars and a total of 67 alleles were amplified. The number of alleles per locus ranged from 1 (Xgwm44) to 7 (Xgwm47), with a mean value of 3.5. The mean of expected heterozygosity ( $\mathrm{He}$ ) ranged from 0.71 (Xgwm149) to 0.27 (Xgwm469). The mean of polymorphism information content (PIC) and the maximum value of Shannon's information index (I) were 0.52 and 0.88 respectively. The number of alleles $(\mathrm{N} a)$, Shannon's information index (I) and mean of expected heterozygosity $(\mathrm{H} e)$, for lines were slightly more than those of cultivars. Average of gene differentiation coefficients (Fst) and gene flow $(\mathrm{Nm})$ for all primers were 0.067 and 6.96 respectively. Analysis of molecular variance (AMOVA) revealed a higher level of genetic variation within lines + cultivars $(89 \%)$ compared to among lines and cultivars $(11 \%)$. Cluster analysis using UPGMA method and simple matching coefficients placed the lines and cultivars in five groups. Similarity coefficients ranged from 0.40 to 1 with a mean value of 0.70 . Some cultivars with the same geographic origin were located in the same cluster. The high level of genetic similarity detected in cultivars may demonstrate the narrow genetic base of Iranian wheat germplasm. However, according to the genetic distance between different groups, lines in divergent groups could be potentially used as parents in wheat breeding programs.
\end{abstract}

Keywords: Bread wheat, SSR markers, Expected heterozygosity, Shannon's information index, Polymorphism Information Content 